\title{
Semidynamical Systems and Hilbert's Fifth Problem
}

\author{
J. W. Neuberger
}

February 4, 2007

\section{Introduction and History}

Semidyanmical system, semiflow, one-parameter semigroup - all the same thing. In this note 'semigroup' is used in place of all three names. Denote by $X$ a Polish space (separable, metric, complete). A semigroup $T$ on $X$ is a function with domain $[0, \infty)$ so that

- $T(0)$ is the identity transformation on $X$.

- $T(t) T(s)=T(t+s), t, s \geq 0$ (indicated product of $T(t)$ and $T(s)$ is composition).

Such a semigroup $T$ is called strongly continuous provided that if $x \in X$ and

$$
g_{x}(t)=T(t) x, t \geq 0 \text {, then } g_{x} \text { is continuous. }
$$

$T$ is called jointly continuous provided that if

$$
g:[0, \infty) \times X \rightarrow X \text { and } g(t, x)=T(t) x, t \geq 0, x \in X, \text { then } g \text { is continuous. }
$$

For many semigroups strong continuity implies joint continuity. A function $g_{x}$ as in (1) is called a trajectory of $T$. If $T$ has domain all of $R$ instead of just $[0, \infty)$ we refer to $T$ as a 'group'.

In his Fifth Problem, Hilbert asked whether assumptions of differentiability that Sophus Lie made were actually a consequence of Lie's algebraic and continuity assumptions. It was shown (see for example[10]) that this was the case. It is less widely recognized that Hilbert raised some additional, rather open ended, questions of a similar nature (see translation in [7], for example). Some of these questions relate to 'functional equations' of which our present semigroups are examples. It seems reasonable to think that Hilbert was interested in algebraic and topological assumptions on a system that imply enough differentiability for an analysis of the system.

The open ended parts of Hilbert's problems will continue to give inspiration for a long time after specific questions are settled. 
Before going further, a reader might keep in mind the following problem associated with Abel's equation: Find functions $f:[0, \infty) \rightarrow R$ so that

$$
f(0)=1, f(x) f(y)=f(x+y), x, y \geq 0 .
$$

If no continuity is specified, there is a tremendous collection of highly discontinuous solutions (think about Hamel bases), but if $f$ is assumed to be continuous and satisfy (2), then $f$ must be differentiable and in fact there must be a number $\alpha$ so that

$$
f(x)=\exp (\alpha x), x \geq 0
$$

Developments over the past seventy years or so for semigroups provide a case study for general aspects of Hilbert's fifth problem. The development started with Marshall Stone's characterization [19] of strongly continuous semigroups (actually Stone had groups) of unitary transformations on a complex Hilbert in terms of infinitesimal generators (the generator is always $i A$ where $A$ is a closed densely defined self adjoint operator and each such transformation 'generates' a unitary semigroup in a the manner of Theorem 1 . Stone's work in this regard is an important mathematical development for quantum mechanics. Stone's theorem has only semigroup and continuity hypothesis. He proves existence of the needed differentiability. In [20], von Neumann studies nonlinear Hamiltonian systems on a Euclidean region $\Omega$ that give rise to representations by means of semigroups (really groups again) of linear transformations defined on $L_{2}(\Omega)$. Von Neumann's paper was an inspiration for the main part of the present note since his Hamiltonian system gives rise to its own semigroup, a nonlinear one.

This note seeks to give some perspective on such semigroups and also to indicate some fairly recent developments. In particular it will be indicated how there is sufficient differentiability available to permit an analysis (without any additional assumptions) of a jointly continuous semigroup $T$ as in the opening paragraph of this note. Those familiar with Riemannian geometry will see a parallel with elements of the present work in that tangent vectors to a Riemannian manifold are means of taking derivatives and Lie generators of this note will be seen as doing the same.

The Hille-Yosida-Philips theorem (see for example [9],[16]), sometimes called the HillePhillips theorem, builds on ideas of Stone. We give a slightly specialized version of this theorem which actually has as a consequence the general version. It is a theorem about linear semigroups $(T(t)$ is a linear transformation for $t \geq 0)$ on $X$ where $X$ is a Banach space. Denote by $Q(X)$ the collection of all closed linear nonexpansive strongly continuous linear semigroups $T$ on $X$ (nonexpansive means that $T(t)$ has norm not exceeding one for $t \geq 0, B$ closed means that $\left\{\left(\begin{array}{c}x \\ B x\end{array}\right): x \in D(B)\right\}$ is closed in $\left.X \times X\right)$. Denote by $G(X)$ the collection of all closed densely defined linear transformations $B$ on $X$ to $X$ so that if $\lambda \geq 0$, then the 'resolvent' $(I-\lambda B)^{-1}$ exists, has domain all of $X$ and is nonexpansive. 
Theorem 1 If $T \in Q(X)$ and

$$
B=\left\{(x, y) \in X \times X: y=\lim _{t \rightarrow 0+} \frac{1}{t}(T(t)-I) x\right\}
$$

then $B \in G(X)$. Moreover, if $B \in G(X)$ then there is $T \in Q(X)$ such

$$
T(t) x=\lim _{n \rightarrow \infty}\left(I-\frac{t}{n} B\right)^{-n} x, t \geq 0, x \in X,
$$

and (3) holds.

Note that if all trajectories of $T$ in the theorem are differentiable, then $B$ is everywhere defined and is consequently bounded thanks to the closed graph theorem. In applications to PDEs, $B$ is generally a differential operator on an $L_{2}$ space and is not defined everywhere.

This theorem indicates how algebraic and continuity conditions imply differentiability given in (3). It illustrates what I call a complete theory in that it gives a collection of semigroups $Q(X)$ and a collection of vector fields $G(X)$ in one-to-one correspondence via constructive rules. For a given $T \in Q(X), B$ is defined by differentiating $T$ at zero. Recovery of a semigroup $T$ from a member $B$ of $Q(X)$ is constructively given by an exponential formula (which to a numerical analyst is inverse Euler's method). How does differentiability on a dense set of trajectories of $T$ arise? Sujppose that $T \in Q(X), x \in X, t>0$ and

$$
y=\frac{1}{t} \int_{0}^{t} T(\cdot) x
$$

Some calculations yield that $y \in D(B)$, the domain of $B$. Since

$$
\lim _{t \rightarrow 0+} \frac{1}{t} \int_{0}^{t} T(\cdot) x=x, x \in X,
$$

$D(B)$ is dense in $X$. This leads to a connection with differential equations since if $y \in D(B)$, the domain of $B$, then the trajectory $u$ starting at $y$ for the corresponding semigroup $T$ satisfies the differential equation:

$$
u^{\prime}(t)=B(u(t)), t \geq 0 .
$$

Trajectories of $T$ which are not differentiable are limits of differentiable trajectories and are often called weak solutions. of (4).

A principle motivation for the present strain of semigroup theory is the identification of classes of time-dependent partial differential equations which may be solved. For many intended applications of linear and nonlinear semigroups, $X$ is a collection of functions and $B$ a differential operator (maybe nonlinear) on a (probably) dense subset of $X$. But 
why semigroups instead of groups? Many systems of time dependent PDEs only have solutions global existing forward in time but not backward in time. Vast collections of time dependent PDEs would be excluded from a theory that dealt with groups rather than semigroups.

Before giving more history of the development of nonlinear semigroups, consider the following example which gives a simple instance of linear representation.

Take $T$ to be the semigroup on $[0, \infty)$ with

$$
T(t) x=t+x, t, x \geq 0
$$

and denote by $C([0, \infty))$ the Banach space of bounded real-value continuous functions on $[0, \infty)$. A corresponding linear representation $S$ of $T$ is given by

$$
(S(t) f)(x)=f(T(t) x)=f(t+x), t, x \geq 0, f \in C([0, \infty)),
$$

a translation semigroup on $C([0, \infty))$. This semigroup $S$ does not fit the HYP theorem in that it is not strongly continuous but it does fit Theorem 2 below. Having this example in mind will help guide one through the history given in the next three paragraphs.

In the late 1950s when I was a graduate student we were studying linear evolution equations such as (4). I asked 'why all this linearity' and in my thesis [11] I took up nonlinear Stieltjes-Volterra integral equations. It was not so long before I settled on nonlinear semigroups, publishing my first results with a nonlinear resolvent in [12] with 'hypothèses très restrictives' according to [1], p-168, (I assumed differentiability that I would have liked very much to prove). The next decade saw an explosion of work on nonlinear semigroups which was, in so far as complete theories are concerned, restricted to strongly continuous nonexpansive (for nonlinear transformations, 'nonexpansive' means Lipschitz norm not exceeding one) semigroups on a convex subset of Hilbert space (see [1],[3],[18] for summeries of this era). In these works, generators are maximal monotone operators, nonlinear analogies to linear generators as $B$ in the HYP theorem. All this work can be characterized as an attempt to create a theory of nonlinear semigroups in analogy to the existing theory. However, Webb in [21] produced an example of a simple-looking nonlinear semigroup on a non-Hilbert Banach space for which the corresponding 'generator' $B$ had very sparse domain, seemingly inadequate for use in recovering the semigroup. This tended to discourage further efforts to develop nonlinear analogues to the established linear theory. In [2] there is a result in which a wide class of nonlinear monotone operators on certain subsets of Banach spaces are shown to give rise to nonexpansive semigrouips, but [2] fails to assign a generator to each such semigroup and hence to circumvent difficulties pointed out by Webb's example.

Around 1970, while reading Sophus Lie's work on construction of integrating factors for ODEs, I was surprised to find that while Lie had (local) nonlinear groups in abundance, 
his 'generators' were linear. What became clear was that his 'generators' were those of an associated linear representation. This led to my trying to apply Lie's eighty year old ideas to nonlinear semigroups, with the aim of getting around obstacles indicated by Webb's example.

Seeing Lie's structure led to my working toward a complete theory of strongly continuous nonlinear semigroups on $X$ in terms of generators of linear representations (acting on a space of functions on $X$ ). I got half of the theory I wanted in [13]. I could start with a nonlinear semigroup $T$ on $X$, derive a generator for a linear representation $S$ of $T$ and even recover $T$ itself from this linear generator. However I wasn't even close to characterizing these generators to get a complete theory. Nothing much more happened in this direction until 1992 at a semigroup conference in Curaçao. During a slow 'problems' session at the conference, I presented my half solution. Bob Dorroh started asking penetrating questions. In a year or so of collaboration, Dorroh and I arrived at results in [4], which are now described, for a complete theory of jointly continuous semigroups on a Polish space.

\section{Semigroups on a Polish Space}

For a Polish space $X$, denote by $S G(X)$ the collection of all jointly continuous semigroups on $X$. Denote by $C B(X)$ the Banach space (under sup norm) of all bounded continuous real-valued functions on $X$. A sequence $\left\{f_{k}\right\}_{k=0}^{\infty}$ in $C B(X) \beta$-converges to $f \in C B(X)$ if the corresponding sequence of sup norms of members of the sequence is bounded and the sequence itself converges to $f$ uniformly on compact subsets of $X$. Denote by $L G(X)$ the collection of all linear transformations $A$ on $C B(X)$ to $C B(X)$ satisfying the following four properties:

- $A$ is a derivation in the sense that if $f, g \in D(A)$, then $f g \in D(A)$ and $A(f g)=$ $f(A g)+(A g) f$.

- $D(A)$ is $\beta$-dense in $C B(X)$.

- If $\lambda \geq 0$ then $(I-\lambda A)^{-1}$ exists, is nonexpansive and has domain all of $C B(X)$.

- If $\gamma>0$ then $\left\{\left(I-\frac{\lambda}{n} A\right)^{-n}: 0<\lambda \leq \gamma, n=1,2, \ldots\right\}$ is uniformly $\beta$-equicontinuous.

Theorem 2 If $T \in S G(X)$ and

$$
A=\left\{(f, g) \in C B(X) \times C B(X): g(x)=\lim _{t \rightarrow 0+} \frac{1}{t}(f(T(t) x)-f(x)), x \in X\right\},
$$

then $A \in L G(X)$. Moreover, if $A \in L G(X)$ then there is a unique $T \in S G(X)$ such that

$$
f(T(t) x)=\lim _{n \rightarrow \infty}\left(\left(I-\frac{t}{n} A\right)^{-n} f\right)(x), x \in X, t \geq 0, f \in C B(X) .
$$


A proof is in [4]. The first part of an argument is relatively routine for those familiar with $[9],[16],[8],[15],[6]$ but will say a few words about the part in which one starts with $A \in L G(X)$ and produces $T \in S G(X)$. First show that $A$ generates a linear semigroup on the norm closure of $D(A)$, then use the fourth condition on $A$ to extend $S$ to all of $C B(X)$. Observe that if $x \in X, \lambda \in[0, \infty)$, then the transformation $\eta$ defined by

$$
\eta(f)=(S(\lambda) f)(x), f \in C B(X)
$$

is a $\beta$-continuous multiplicative linear transformation from $C B(X)$ to $R$ (its null space is thus a maximal ideal in $C B(X))$ and that happily $\eta$ is given by point evaluation, i.e., there is $y_{x, \lambda} \in X$ so that

$$
\eta(f)=f\left(y_{x, \lambda}\right), f \in C B(X) .
$$

Then take

$$
T(\lambda) x=y_{x, \lambda}
$$

and check that $T$ is indeed a jointly continuous semigroup on $X$ whose Lie generator is $A$. The fact that real-valued $\beta$-continuous multiplicative linear functions on $X$ are given by point evaluations is precisely the reason that separability is required for $X$ in the theorem (see [17],[4] for details).

\section{Related Semigroups on Spaces of Measures}

Given $T \in S G(X)$, there is a second linear semigroup even more closely related to $T$. Denote by $M(X)$ the set of compact regular Borel measures on the Borel sets $B(X)$ of $X$ and define the linear semigroup $U$ on $M(X)$ by

$$
(U(t) \mu)(\Omega)=\mu\left\{T(t)^{-1} \Omega\right\}, t \geq 0, \mu \in M(X), \Omega \in B(X) .
$$

Following [14], one has that $U$ is the semigroup adjoint to $S$ (see [5], for example):

$$
(S(t) f)(x)=f(T(t)), x \in X, t \geq 0, f \in C B(X)
$$

in the sense that

$$
U(t)=S(t)^{*},
$$

with the following understanding: $S$ may be regarded as a semigroup on $C B(X)$ with the $\beta$ - topology. Members of the dual of $C B(X)$, under the $\beta$-topology, may be represented by integration with respect to compact regular measures. Using this dual space, $S(t)^{*}, t \geq$ 0 , may be defined and it turns out that (5) holds (see [17]).

The semigroup $U$ has the property that if $x \in X$, and $\delta_{x}$ is the Dirac measure associated with $x$, then

$$
U(t) \delta_{x}=\delta_{T(t) x}, t \geq 0
$$


In a sense, $U$ is a linear extension of the nonlinear semigroup $T$ if one identifies $X$ with Dirac measures on $X$. In summary, start with $T$, take its linear representation $S$, take the dual semigroup $U$ os $S$, find out that $U$ is a linear extension of $T$. What has happened can be roughly described as this: points of $x \in X$ are first identified with corresponding Dirac measures concentrated at $X$. Then more measures, compact regular measures, are introduced to keep company with the Dirac measures. The resulting measure space has enough elements to provide a generator, defined by differentiation, in terms of which $T$ can be recovered, thus finding another way around the difficulties associated with Webb's example.

\section{Possible Application}

Close relationships of semigroups $U$ and $S$ in the previous section with Kolomogoroff's forward and backward equations of stochastic differential equations can be seen (see [5] for example). This structure helped me write a code for a fully nonlinear filter, but that is another story.

A word about other possible applications. For $X$ a subset of a Banach space, and $T \in S G(X)$, suppose that $T$ has a densely defined generator $B$ in the conventional sense and Lie generator $A$. That is,

$$
B x=\lim _{t \rightarrow 0+} \frac{1}{t}(T(t) x-x), x \in D(B) .
$$

If $f \in C B(X)$ is a $C^{1}$ function in $D(A)$, then

$$
(A f)(x)=f^{\prime}(x) B x, x \in D(B) .
$$

Sophus Lie would recognize (7) right away. However, members of $D(A)$ need not be Fréchet differentiable. One has only that if $f \in D(A)$, then

$$
(A f)(x)=\lim _{t \rightarrow \infty} \frac{1}{t}(f(T(t) x)-f(x), x \in X .
$$

Even if $x \in D(B)$ one gets only that $(A f)(x)$ is a directional derivative of $f$ in the direction $B x$ with Fréchet differentiability probably not holding. So even though (7) suggests a form for a Lie generator of $T$ in this case, it is still not clear how to articulate precisely the nature of $D(A)$. Nevertheless, I offer the following conjecture in which I don't have particular confidence, but rather hope it might serve to suggest other more reasonable speculation.

Conjecture. Suppose $X$ is an open subset of a Banach space $Y$ and $B$ is a closed densely defined transformation on $X$ to $Y$. Denote by $A$ the collection of all $(f, g) \in$ $C B(X)^{2}$ so that

$$
g(x)=\lim _{t \rightarrow \infty} \frac{1}{t}(f(x+t B x)-f(x)), x \in D(B)
$$


If $A$ satisfies the four conditions preceding Theorem 2, then there is a unique semigroup $T$ on $X$ with conventional generator $B$ as in (6) and Lie generator $A$.

I feel quite certain that in any event, Theorem 2 will not be easy to apply in the sense that it is going to be fairly difficult to show how given concrete examples fit the hypothesis. I am reminded of Ralph Phillips' comment to me: 'It is not easy to apply semigroup theory to differential equations.'

\section{Final Comment}

In the summer of 2005 I greatly profited from a stay at the Max Planck Institute in Leipzig. I was asked to give at talk in Sophus Lie Raum. That I didn't sieze the chance to talk about the present topic is a matter of some regret.

\section{References}

[1] H. Brezis, Operateurs maximaux monotones, North Holland, 1973.

[2] M. Crandall and T. Liggett, Generation of semi-groups of nonlinear transformations on general Banach spaces, Amer. J. Math. 93 (1971), 265-298.

[3] G. da Prato, Applications croissantes et èquations d'évolutions dans les espacè de Banach, Academic Press 1976.

[4] J.R. Dorroh and J.W. Neuberger, A theory of strongly continuous semigroups in terms of Lie generators, J. Functional Analysis 136 (1996), 114-126.

[5] E.B. Dynkin, Markov Proceses-I, Grund. Math. Wiss. 121, Springer 1965.

[6] K.-J. Engel and R. Nagel, One-parameter semigroups for linear evolution equations, Springer 1999.

[7] http://aleph0.clarku.edu/ djoyce/hilbert/problems.html

[8] J. Goldstein, Semigroups of linear operators and applications, Oxford 1994.

[9] E. Hille and R. Phillips, Functional analysis and semigroups, American Mathematical Society, 1957. 
[10] D. Montgomery and L. Zippin, Topological Transformation Groups, Interscience Publishers, New York. London (1955).

[11] J.W. Neuberger, Continuous Products and Nonlinear Integral Equations, Pac. J. of Math., 8 (1958), 529-549.

[12] J.W. Neuberger, An exponential formula for one-parameter semigroups of nonlinear transformations, J. Math. Soc. Japan 18 1966, 154-157.

[13] J.W. Neuberger, Lie Generators for One Parameter Semigroups of Transformations, J. Reine Ang. Math., 258 (1973), 133-136.

[14] J.W. Neuberger, A Complete Theory for Jointly Continuous Nonlinear Semigroups on a Complete Separable Metric Space, J. Applicable Analysis 78 (2001), 223-231.

[15] A. Pazy, Semigroups of Linear Operators and Applications to Partial Differential Equations, Appl. Math. Sci. 44, Springer, 1983.

[16] F. Riesz and Sz.-Nagy, Functional Analysis, Ungar 1965, (Dover 1990).

[17] D. Sentilles, Bounded continuous functions on a completely regular space, Trans. American Math. Society 168 (1972), 311 - 336.

[18] R.E. Showalter, Monotone operators in Banach spaces and nonlinear partial differential equations, Amer. Math. Soc. Math. Sur. Monographs 49, 1996.

[19] M. H. Stone, On one-parameter unitary groups in Hilbert Space, Annals of Mathematics 33 (1932), 643-648.

[20] J. Von Neumann, Dynamical systems of continuous spectra, Proc. Nat. Acad. Sci 18 (1932), 278-286.

[21] G.F. Webb, Representation of semigroups of nonlinear nonexpansive transformations in Banach spaces, J. Math. Mech. 19 (1969/70), 159-170. 